\title{
Recent advance in polymer solar cells: enhancing power conversion efficiency over $10 \%$ with deterministic aperiodic nanostructures
}

Polymer solar cell (PSC) is a promising photovoltaic technology for clean and renewable energy sources owing to its mechanical flexibility and large-area, low-cost manufacturing compatibility. Significant progress in device performance has been made in the recent years through the development of new materials, morphology control in photo-active layer, interface engineering in multi-layer device, and device fabrication processes. However, further improvement in efficiency exceeding $10 \%$ in single-junction PSCs remains a daunting challenge for commercial applications owing to the compromise between optical absorption and charge transport in the active layer.

The recent breakthrough made by the collaboration between Jian-Xin Tang's group and Yongfang Li's group at Soochow University was published in Advanced Materials on Nov. 18, 2014 [1]. The researchers developed a universal method for the optical manipulation of light by integrating a deterministic periodic nanostructures (DANs) into the real devices through soft nanoimprint lithography. The DANbased light trapping scheme can effectively enhanced broadband light harvesting and provided optimum charge extraction simultaneously, leading to a substantial increase in power conversion efficiency and an overall efficiency exceeding $10 \%$ for single-junction PSC from a blend of poly[4,8-bis(5-(2-ethylhexyl)thiophen-2-yl)benzo[1,2- $b$ :4,5$\left.b^{\prime}\right]$ dithiophene-co-3-fluorothieno[3,4- $\left.b\right]$ thiophene-2-carboxylate]:[6,6]-phenyl-C71-butyric acid methyl ester (PTB7$\left.\mathrm{Th}: \mathrm{PC}_{71} \mathrm{BM}\right)$ as the photoactive layer. Experimental work and theoretical simulations reveal that the performance enhancement can be ascribed to the self-enhanced absorption owing to collective effects, including the pattern-induced anti-reflection, light scattering as well as surface plasmonic resonance, together with a reduced recombination loss. The two-dimensional sub-wavelength DANs with a continuously tapered morphology on the patterned surface provide a superior gradient refractive index profile at the interface, which can significantly manipulate the incident light in all azimuthal directions over the entire solar spectra.
This work is fundamentally important in that the authors proposed and demonstrated a route to controlling the light harvesting in PSC from various material systems with different bandgaps and the compatibility for large-area and low-cost manufacturing. This work is expected to promote rapid development of high-efficiency PSC and other organic optoelectronic devices with new device structures. Other research areas might benefit from this work as well. For example, the light out-coupling efficiency of organic lightemitting diodes (OLEDs) is limited as low as $\sim 20 \%$ because of the optical confinement and internal reflection by the high refractive index of organic materials and transparent substrate. The DANs developed here may lead to a drastic boost in light out-coupling and can effectively enhance the device performance of OLEDs.

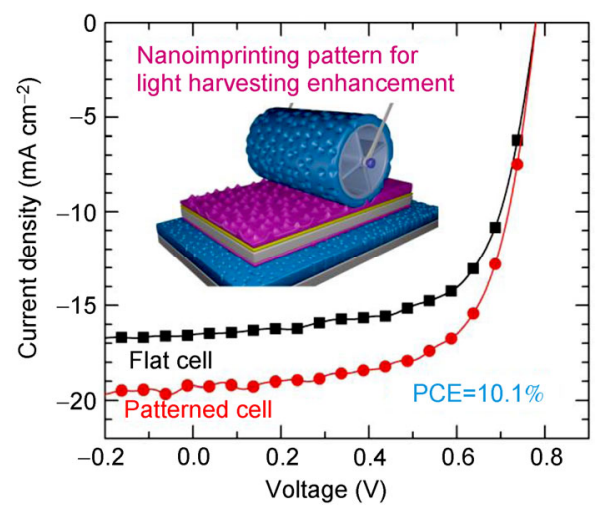

Figure 1 Illustration of the preparation and the device performance of a single-junction PSC containing the dual-sided nanoimprinted DANs.

Hongbin $\mathrm{Wu}$ Institute of Polymer Optoelectronic Materials and Devices, State Key Laboratory of Luminescent Materials and Devices, South China University of Technology

1 Chen JD, Cui C, Li YQ, Zhou L, Ou QD, Li C, Y. Li, Tang JX. Single-junction polymer solar cells exceeding $10 \%$ power conversion efficiency. Adv Mater, 2014, DOI: 10.1002/adma.201404535 